\title{
SPACE OBSERVATIONS NEEDED FOR IMPROVING OUR KNOWLEDGE OF SOLAR FLARES
}

\author{
A. B. SEVERnY \\ (Crimean Astrophysical Observatory, U.S.S.R.)
}

1. Up to the present time the technical possibilities of space research were not quite adequate for obtaining the ultraviolet spectrum of flares that occupy only a small portion (less than $10^{\prime \prime}$ of arc) of the solar disk. Up to now all observations have been made in integrated sunlight. The importance of the knowledge of UV spectrum of flares is hardly necessary to emphasize. For example we just could mention that if ultraviolet spectra were available for a flare say from $850 \AA$ up to $2000 \AA$, we would be able to estimate such extremely important parameters as the number of hydrogen atoms $\mathrm{N}_{1}$ in the first quantum state (by using $\mathrm{L}-\alpha, \mathrm{L}-\beta$, etc.), electronic temperature and density (from Lyman continuum) and other physical parameters of a flare which at the present time we try to derive by different indirect and inadequate methods. Several resonance (ultimate) lines are concentrated in this spectral interval and their careful examination in flares can bring additional important information about conditions prevailing in flares and in the underlying chromosphere. The same applies of course to the whole UV spectrum below $800 \AA$, and in particular to the resonance lines of HeI $(\lambda 512 \AA)$ and HeII $(\lambda 304 \AA)$, as well as to a number of lines of highly ionized atoms.

The purpose we are discussing should be to put forward before space-research technique a requirement to improve the pointing and guiding of spacecraft to secure the pointing with an accuracy of several seconds of arc during the lifetime of a flare. Probably this requirement cannot be solved without manned space flights for final adjustment and correction of the position of a flare on the slit of an UV spectrograph. Until we are able to get an UV spectrum of any interesting small area of the solar disk with the aid of spacecraft, we are far from any solution of the most important problems relating to the physics of solar activity. The task under consideration (accurate pointing) is not hopeless, especially for such a bright object as our Sun, and we hope that the accuracy of even unmanned pointing of $\sim 1^{\prime \prime}$ could be considered as feasible in the nearest future.

However, these space observations (probably with small dispersion $\sim 3-5 \AA / \mathrm{mm}$ as a first step) should be accompanied by simultaneous ground-based observations including (as a minimum program) the spectrograms of flare in the visible spectrum in the course of the whole flare development, as well as $\mathrm{H} \alpha$ and $\mathrm{K}$ direct photography of the process.

Perek (ed.), Highlights of Astronomy, 530-532. (O I.A.U. 
2. The information on flares in integrated sunlight which we can obtain by using spacecraft (rockets and satellites) is of course still important, despite the fact that the contribution to this emission of a flare, by area, is no more than $0.1 \%$. We think that it is important to know the time variation of the $\mathrm{L}-\alpha$ and $\lambda 304$ emissions during important flares, provided we can evaluate adequately the energy excess due to a flare from these data. Also valuable would be the information on the variations of the Lyman continuum during the flare process and other active processes, if these variations could be detected at all. In this connection I would like to mention the recent successful experiment realized by Ing. Bruns of the Crimean Observatory, who found some variations in the profile of Lyman continuum as recorded photoelectrically during periods of enhanced solar activity in July this year. The photoelectric scanning spectrometer was working for $\sim 20$ days during the flight of Sputnik Cosmos- 166 . The analysis of the telemetric records is now in progress, and we hope to get some important information about the possible changes of the electron temperature of the chromosphere in connection with the varying solar activity.

3. The space observations of X-ray emission indicate that sometimes $\mathrm{H} \alpha$ flares are not accompanied by $\mathrm{X}$-rays, and some $\mathrm{X}$-ray bursts do not seem to have counterparts in the optical region. (These indications have been obtained mainly during quiet periods on the Sun.) In principle the processes leading to $\mathrm{X}$-rays take place in the corona and this is the reason why they may remain unnoticed by $\mathrm{H} \alpha$ patrol of solar flares. But we should not exclude the possibility that such violent and impulsive processes as 'moustaches' or bombs could also be a source of X-rays. Moustaches are extremely transient phenomena, their lifetime varies (in the mean) form several minutes to $20 \mathrm{~min}$. At the same time short-lived bursts of X-rays of similar duration are frequently observed during the period of enhanced activity and, in particular, they have been recorded by Dr. Kreplin during the period preceding the proton flare of July 7 , when moustaches appeared. Therefore, we think that the on- and off-band observations of moustaches combined with spacecraft records of X-rays would be desirable, because they could shed some light on the origin of small bursts of $X$-rays. At the usual on-band solar $\mathrm{H} \alpha$-patrol these processes (moustaches) are likely to remain unnoticed.

If X-ray emission is partly due to bremsstrahlung we will probably have some continuation of this emission in the visible part of spectrum, and we found several years ago that observed continuous visible spectrum of moustaches could be considered as a continuation of the bremsstrahlung spectrum of electrons. In any case, one of the most important problems of X-ray space investigations of flares would be to derive the course of continuous emission of flare in the X-ray and UV region. We still do not have adequate observations, although there are some indications on the emission of thermal origin at the temperatures $\simeq 10^{7} \mathrm{~K}$. Still there are some very short impulsive bursts of X-rays, the origin of which is difficult to attribute to some thermal source. 
4. For our knowledge of how a flare could be initiated, it would be important to have very accurate time marking for both the space and the ground-based observations (in particular $\mathrm{H} \alpha$-solar patrol). At the present time the keeping of time for solar observations deserves improvement at most of the observatories. The adequate comparison of the time of onset of $\mathrm{H} \alpha$ flare and X-ray bursts could be done only if we have precise time markings. In principle it is not excluded that the process in the X-ray region can precede the process observed in the visible spectrum, especially for flares with a rapid, impulsive growth of $\mathrm{H} \alpha$ intensity. There are some indications that small $\mathrm{X}$-ray bursts precede the optical $\mathrm{H} \alpha$ flare, as pointed out by Mandelštam. If accelerated particles appear during these bursts, we should conclude that the optical flare phenomenon is related to the supply of high-energy protons and other particles, which produce a high degree of ionization and excitation of atoms.

5. The X-ray space observations show that the area responsible for $\mathrm{X}$-ray emission is small, not larger than $0.1 \%$ of solar disk $\left(<30^{\prime \prime}\right)$. I do not think we would be surprised if further observations show that this area is as small as the fine structure of an active region, or that the $\mathrm{X}$-ray emission as well as the emission in optical range is concentrated mainly in small grains and filaments with characteristic size $\leqslant 1$ ". The mottled structure of the Sun in $\lambda 304 \AA$ and fine structure of $L-\alpha\left(\sim 2^{\prime \prime}-3^{\prime \prime}\right)$ are probably first indications to that effect. Moreover, for the understanding of the flare process it would be important to know whether the X-rays emitting area coincides with the area responsible for the visible spectrum. All these problems could probably be solved if we can secure a resolution of the order of $1^{\prime \prime}$, for direct image X-ray photographs of the Sun. As far as we know such resolution is about to be realized in the space observations in the nearest future.

Without being complete, these are just some comments on possible observations relating to solar flares. 